\title{
An Investigation into the Fault Diagnosis Technology of Warships
}

\author{
Wu Yan-bin*, Yao Lu, Yang Jianbin \\ Dept. of Management Science, Naval Univ. of Engineering \\ Wuhan, China
}

\begin{abstract}
This paper elaborates the connotation of the fault diagnosis theory, proposes several fault diagnosis technologies and their applications to naval ships, and explores the developing direction of fault diagnosis technology.
\end{abstract}

Key Words- fault diagnosis; neural network; expert system

\section{I . INTRODUCTION}

Nowadays, it has become a hot domain with the increasing development of the fault diagnosis. The fault can be regarded as a abnormal phenomena of an arbitrary system and have no expected feature when a system breaks down. The fault diagnosis technology mainly includes three aspects: fault detection, fault isolation and fault identification. Fault detection means to judge whether the system breaks down or not and the accurate time of the fault. Fault isolation means to ensure the position and type of the fault after fault detection is found. Fault identification means to confirm the fault's size and its time-variant characteristic after making fault isolation. Therefore, the fault diagnosis technology is to solve the problems of pattern classifications and identifications, that's to say, the system is divided into normal and abnormal operating state, while what kind of fault the abnormal sample on earth belongs to is a matter of mode recognition. With the development of the fault diagnosis technology in recent decades, the researches in this field have been carried out widely and deeply.

This paper discusses the fault diagnosis methods and their applications on ships based on knowledge. The fault diagnosis methods can be divided into three categories. firstly, method totally based on signal processing; secondly, method based on analytical model; and thirdly, method based on knowledge. The signal processing method needs to add simultaneously detection means and calculated amount, so that it make use of signal model to analyze the detected signals directly. Analytical model method can be divided into state estimation, parameter estimation and equivalent spatial method. The detected information is processed according to the corresponding mathematical method, which is based on the diagnosis object of mathematical model, but this kind of method is difficult to obtain more accurate object mathematical model. While the artificial intelligence and computer technology develop fast, meanwhile it also provides new theoretical basis for the fault diagnosis technology, and the new fault diagnosis methods are also developed. Its advantage lies in having "intelligent” features and don't need precise mathematical model. The method based on knowledge can be divided into the following types: the fault diagnosis method of expert system, fault diagnosis method of fault tree, fault diagnosis method of neural network, fault diagnosis method of fuzzy as well as fault diagnosis method of data fusion, etc.

\section{THE FAULT DIAGNOSIS METHOD BASED ON ARTIFICIAL NEURAL NETWORK}

The fault diagnosis method based on artificial neural network actually is to build up the reference model of object by using neural net, its working principle as shown in the Fig. 1. As reference model of artificial neural ANN1, it actually operates with real system in 
parallel. The output acts as the anticipated output â of the real system, then a residual error is arose compared with the real system . Once more to take advantage of artificial neural network ANN2 to carry out residual analysis, complete feature extract, fault detect and separation.

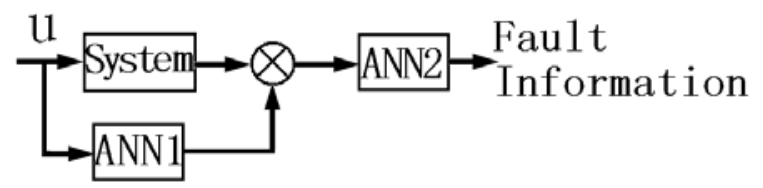

Fig. 1 The working principle of fault diagnosis based on artificial neural network

\section{THE FAULT DIAGNOSIS METHOD BASED ON SELF- ADAPTIVE FUZZY LOGIC SYSTEM}

The self-adaptive fuzzy logic system has learning capability of fuzzy logic system. It is composed of a series of "IF, THEN" rules, so all the described information which adopts fuzzy "IF, THEN" formal language in this system can be used, the describing data information adopted "input-output" data can be made use of by self-adaptive fuzzy logic system through learning algorithm. It means that the system can unite the knowledge and data information described by language, its working principle is founded on the fault diagnosis method based on self-adaptive fuzzy logic system as shown in the Fig. 2.

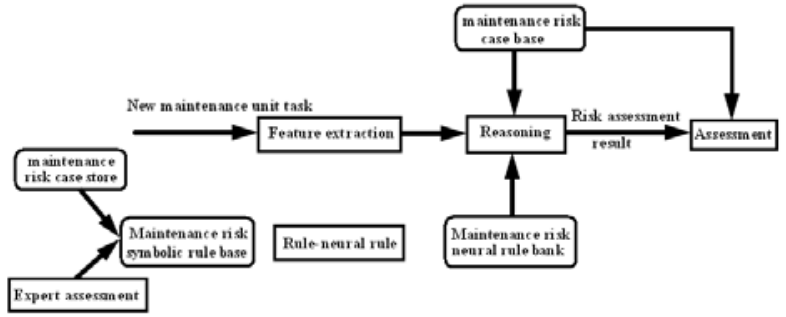

Fig. 2 The working principle of fault diagnosis based on self-adaptive fuzzy logic system

\section{THE INTELLIGENT FAULT DIAGNOSIS METHOD BASED ON EXPERT SYSTEM}

With development of computer science and artificial intelligence, the intelligent fault diagnosis method based on expert system can overcome overdependence on model of the fault diagnosis method based on model, and the intelligent fault diagnosis method is especially effective to the fault diagnosis of complicated system, and has been widely used in many fields. The block diagram of the intelligent fault diagnosis method based on expert system is as shown in the Fig. 3.

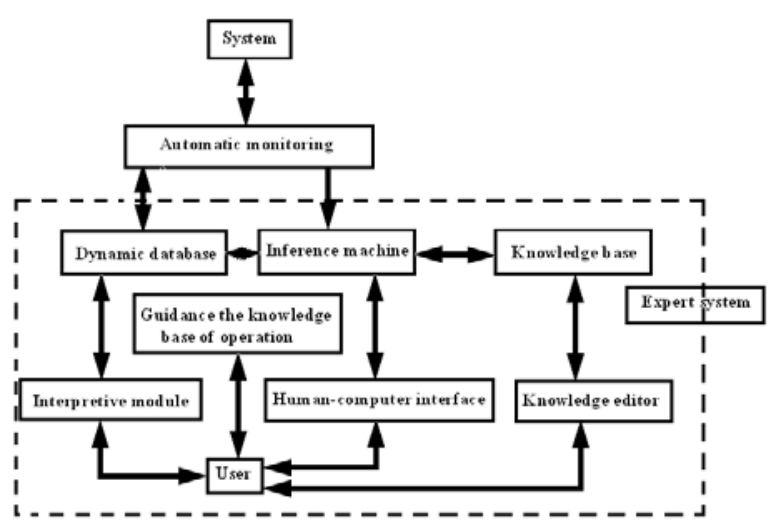

Fig. 3 The real-time supervision and fault diagnosis system based on expert system

An integrated expert system is composed of inference machine, knowledge acquisition module and interpretive module, which contains knowledge base store and manages expert knowledge. Expert knowledge includes the whole professional field knowledge, comprehension of special issues and skills for solving the related problems. The primary problem of expert system is the acquisition and expression of knowledge, expert system based on computer needs to collect sufficient expert knowledge, and store it into knowledge base. Inference machine is a mechanism of coordinating and controlling the whole system, it will solve the current problems according to the facts and rules of repository by some reasoning strategies, of course the conclusion drawn by the expert system should be accordant with domain experts. Knowledge acquisition module focuses on how to transform experts' experienced knowledge into the knowledge that computer can use and interpret.

The accuracy of the fault diagnosis relies on experts’ knowledge and level of expertise knowledge 
base, the more knowledgeable and more experienced the expert knowledge is, the more efficiency and more reliable the knowledge base will be. Otherwise, the "conflict resolution" and "combination explosion" problems will take place, which can lead to the reasoning inefficiency of expert system.

\section{CONCLUSION}

The artificial neural network has self-learning, selfadaptive, robustness and fault tolerance, so it can deal with the information and non-linearity matter of fuzzy, random, incompatible features, and it also has the capability of associative memory, and is suit for some problems of fault diagnosis. But the artificial neural network needs a lot of training samples, which is difficult to gain in the fault diagnosis. Therefore, to acquire enough representative samples requires further research. In addition, the study of network model and learning algorithm need to deepen further in order to solve the more complicated fault problems.

\section{REFERENCES:}

[1] Ye Yinzhong, Pan Rifang, "The fault detect and diagnosis methods," [J]. Information and Detect. 1995,14(6): 27-34.

[2] Xuan Jianping, Zhang Hua, "The Review on Theory and Methods of Fault Detect and Diagnosis Based on Dynamic Model," [J].Journal of Vibration Engineering. 2010,17(2):153-158.

[3] Zhang Ping, Zhou Donghua, "The Application and Approach for Fault Diagnosing of Dynamic System," [J]. 2012,17(2):123-125.

[4] Tang $\mathrm{T}$, Liu $\mathrm{Y}$ et al. ANN, Based fault diagnosis method with a combined BP algorithm [C]. Proceedings of UKACC International Conference on Control,1996.

[5] Tianhao Tang, et al. A, “ Fuzzy and Neural Network Integrated Intelligence Approach for Fault Diagnosing and Monitoring," [C]. Proceedings of the 1998 UKACC International Conference on Control, Swansea, UK, 1998. 